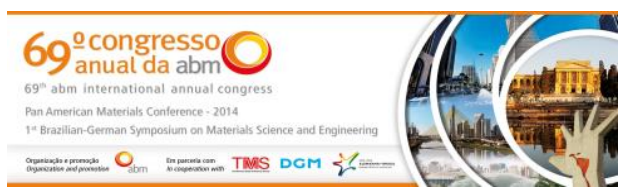

Tema: Lavra e Tratamento de Minério

\title{
IMPACTO DA QUALIDADE DOS DADOS NO PLANEJAMENTO DE CURTO PRAZO*
}

\section{Resumo}

Cristina da Paixão Araujo ${ }^{1}$ Marcel Antônio Arcari Bassani² João Felipe Coimbra Leite Costa ${ }^{3}$

O planejamento de lavra de curto prazo tipicamente confia em amostras obtidas de furos de desmonte em uma operação de lavra a céu aberto. Essas amostras podem conter grande erro de amostragem. O objetivo deste artigo é avaliar o impacto do erro de amostragem na recuperação de lavra. Para comparação, foi utilizado banco de dados Walker Lake exaustivo considerado como a distribuição real dos dados. A metodologia consiste na obtenção de amostras a partir dos dados exaustivo, em um espaçamento regular de $5 \times 5 \mathrm{~m}$. Em seguida, o erro de amostragem foi adicionado às amostras. Posteriormente, as amostras foram utilizados para estimar os blocos e classifica-los como minério ou estéril. Quatro cenários com amostras com $0 \%, 10 \%$, $20 \%$ e $30 \%$ de erro relativo. A recuperação de lavra de cada cenário é comparada com a real distribuição dos dados. Além disso, o erro de classificação do bloco é avaliado para os quatro casos. Os resultados mostram que problemas na amostragem e no protocolo de preparação de amostras pobres afetam a curva teor tonelagem e a classificação dos blocos em minério e estéril.

Palavras-chave: Erro de classificação; Erro de amostragem.

\section{Abstract}

\section{IMPACT OF DATA QUALITY ON SHORT TERM PLANNING}

Short-term mine planning typically relies on samples obtained from blastholes in an open pit operation. These samples may carry a large sampling error. The aim of this article is to evaluate the impact of the sampling error in mining recovery. For comparison purposes, the exhaustive Walker Lake was used and it is considered to be the true grade distribution. The methodology consists in obtaining samples from the exhaustive data set at a regular spacing of $5 \times 5 \mathrm{~m}$. Then, a sampling error is added to the samples. Afterwards, the samples were used to estimate the blocks and decide whether they are ore or waste. Four scenarios were evaluated: samples with $0 \%, 10 \%$, $20 \%$ and $30 \%$ of relative error. The mining recovery of each scenario is compared with the true block grade distribution. Also, the block misclassification is assessed for the four cases and the results show that the problems in sampling and preparation protocol affect the grade tonnage curve and the block misclassification in the ore and waste.

Keywords: Misclassification; Error sampling.

1 Química, Estudante de Mestrado, Departamento de Engenharia de Minas, Universidade Federal do Rio Grande do Sul (UFRGS), Porto Alegre, RS, Brasil.

2 Engenheiro de Minas, Estudante de Mestrado Departamento de Engenharia de Minas, UFRGS, Porto Alegre, RS, Brasil.

3 Engenheiro de Minas, Phd, Professor Associado, Departamento de Engenharia de Minas, UFRGS, Porto Alegre, RS, Brasil.

* Contribuição técnica ao 69 Congresso Anual da ABM - Internacional e ao 14ํㅡㄹ ENET - Encontro Nacional de Estudantes de Engenharia Metalúrgica, de Materiais e de Minas, 21 a 25 de julho de 2014 , São Paulo, SP, Brasil. 


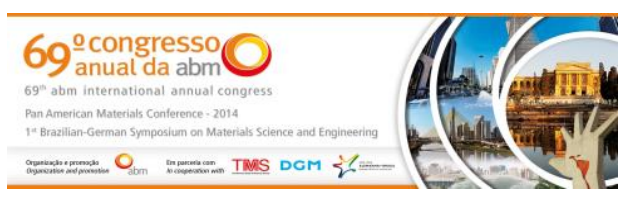

\section{INTRODUÇÃO}

As amostras de desmonte utilizadas no planejamento de lavra de curto prazo são utilizadas na estimativa de blocos cujos valores definem se serão classificados como blocos de minério ou estéril. Os teores dessas amostras estão sujeitos a erros provenientes da amostragem e preparação de amostras. Os resultados dessas amostras são utilizados para aprimorar a tomada de decisões e classificar o recurso como minério ou estéril. Geralmente os protocolos de preparação destas amostras possuem erros que podem ocasionar perdas devido à classificação errônea do recurso.

Por convenção, estabelece-se que $80 \%$ da variância do erro global da estimativa está relacionado à amostragem, $15 \%$ à preparação de amostra e somente $5 \%$ corresponde a análise química das amostras. O erro analítico é pequeno comparado ao erro total de amostragem, no entanto ele influenciará na estimativa global da estimativa, que corresponde à soma do erro total de amostragem (TE) com o erro analítico (AE) [1-3].

De acordo com a Teoria de Amostragem de Gy [4], os erros de amostragem podem ser decompostos em erro fundamental (FE), erro de segregação e agrupamento, erro de delimitação, erro de extração e erro de preparação. O erro fundamental ocorre devido à distribuição irregular partículas no minério e pode ser quantificado. Os outros três tipos de erro são difíceis de quantificar, mas podem ser minimizados através da manutenção de boas práticas de controle de qualidade para reduzir o seu impacto potencial $\mathrm{O}$ erro fundamental depende da constante de amostragem (que quantifica a heterogeneidade do lote em cada estágio de pulverização), o tamanho das partículas (D95), o peso do lote e da amostra retirada a partir dele. Em um protocolo de preparação de amostras, o erro fundamental (FE) é adicionado em cada etapa onde ocorre a redução de massa da amostra. Este é aditivo ao longo do protocolo. A quantificação deste erro pode ser utilizada para avaliar a qualidade do protocolo amostral, quando o erro associado ao erro fundamental (FE) é inferior a 10\% este pode ser considerado aceitável e acima de $30 \%$, este protocolo não é aceitável.

A presença de erros aumenta a variância dos dados impactando no modelamento do variograma dos dados. Modelos incorretos podem levar a erros a substanciais na estimativa e na classificação de blocos que podem ser refletidos em perdas significativas para o lucro operacional.

O presente trabalho avalia o impacto dos erros incorporados nos dados a partir da amostragem e preparação das amostras de desmonte no planejamento de curto prazo utilizadas no SMU (unidade seletiva de mina) para classificação dos blocos em minério e estéril.

\section{MATERIAIS E MÉTODOS}

\subsection{Estatística Descritiva do Banco de Dados Exaustivo}

O banco de dados exaustivo, utilizado para este estudo, é conhecido como Walker Lake [5], é composto por dados bidimensionais em uma área de $260 \times 300$ metros, dispostos na malha regular de dados em $1 \times 1 \mathrm{~m}$ totalizando 78000 dados pontuais. Para este estudo, a variável $\mathrm{V}$ foi reescalada para se assemelhar a um depósito de cobre. Para obter o cenário considerado como a distribuição real do depósito, os

* Contribuição técnica ao 69ํ Congresso Anual da ABM - Internacional e ao 14 ENEMET - Encontro Nacional de Estudantes de Engenharia Metalúrgica, de Materiais e de Minas, 21 a 25 de julho de 2014 , São Paulo, SP, Brasil. 


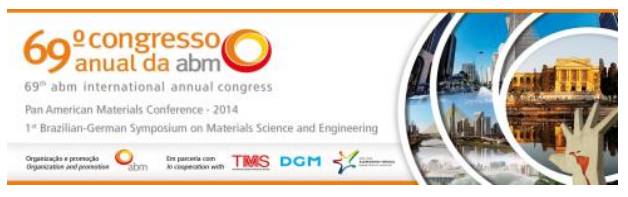

dados pontuais foram transformados para blocos com espaçamento regular de 5,0 $\mathrm{x}$ $5,0 \mathrm{~m}$ totalizando 3120 blocos.

\subsection{Adição do Erro}

Este estudo é composto por quatro cenários distintos, o primeiro não possui erros de amostragem e preparação de amostras. Esse foi obtido a partir da estimativa do bloco por krigagem ordinária [6]. Os demais cenários possuem erros relativos distintos de $10 \%, 20 \%$ e 30\%. O erro aleatório relativo foi adicionado (ou subtraído), assumindo uma distribuição Gaussiana com média zero e desvio padrão determinado pelo produto do erro relativo multiplicado pelo teor do bloco. Esses dados representaram as amostras de desmonte utilizadas no curto prazo.

A Tabela 1 mostra a estatística descritiva dos dados considerados reais, sem erro e do conjunto de dados com $10 \%, 20 \%$ e $30 \%$. Todos os conjuntos de dados possuem médias próximas ao valor da média real o que indica a inexistência de viés [7].

Tabela 1. Dados perturbados com erro utilizados para estimativa utilizando krigagem ordinária

\begin{tabular}{lcccc}
\hline Dados & Média & Variância & $\begin{array}{c}\text { Desvio } \\
\text { Padrão }\end{array}$ & CV \\
\hline Real & 2,77 & 6,24 & 2,49 & 0,90 \\
\hline Erro_0\% & 2,78 & 6,20 & 2,48 & 0,89 \\
\hline Erro_10\% & 2,77 & 6,20 & 2,48 & 0,89 \\
\hline Erro_20\% & 2,78 & 6,81 & 2,60 & 0,93 \\
\hline Erro_30\% & 2,79 & 7,50 & 2,73 & 0,97 \\
\hline
\end{tabular}

\subsection{Continuidade Espacial e Estimativa dos Teores}

A continuidade espacial é relação entre pontos separados por uma distância $\boldsymbol{h}$. O variograma, definido por $\mathbf{2} \boldsymbol{\gamma}(\boldsymbol{h})$, permite representar quantitativamente a variação de um fenômeno regionalizado no espaço, ou seja representar a diferença da variância entre os pontos por uma distância $\mathrm{h}[6,8]$.

$$
\operatorname{Var}\{Z(x)-Z(x+h)\}=E=\{[Z(x)-Z(x+h)]\}^{2}=2 \gamma(h)(1)
$$

Para representação do fenômeno foi utilizado o semi-variograma, $\boldsymbol{\gamma}(\boldsymbol{h})$, que representa a metade do variograma definido por:

$$
\gamma(\boldsymbol{h})=\left(\boldsymbol{E}=\{[\boldsymbol{Z}(\boldsymbol{x})-\boldsymbol{Z}(\boldsymbol{x}+\boldsymbol{h})]\}^{2}\right) / 2
$$

Os modelos semivariográficos são caracterizados por quatro componentes (Figura 1): alcance (a) que corresponde a distância dentro da qual as amostras apresentamse correlacionadas espacialmente, o patamar $(\boldsymbol{C})$ que é o valor do semivariograma correspondente a seu alcance (a), a partir dele considera-se que não existe mais dependência espacial entre as amostras, porque a variância da diferença entre pares de amostras $(\boldsymbol{V} \boldsymbol{a r}[\boldsymbol{Z}(\boldsymbol{x})-\boldsymbol{Z}(\boldsymbol{x}+\boldsymbol{h})])$ torna-se invariante com a distância, o efeito pepita $\left(\boldsymbol{C}_{\mathbf{0}}\right)$ que mostra a descontinuidade do semivariograma para distâncias menores do que a menor distância entre as amostras e parte desta descontinuidade

* Contribuição técnica ao 69 Congresso Anual da ABM - Internacional e ao 14ํㅡㄹ ENET - Encontro Nacional de Estudantes de Engenharia Metalúrgica, de Materiais e de Minas, 21 a 25 de julho de 2014 , São Paulo, SP, Brasil. 


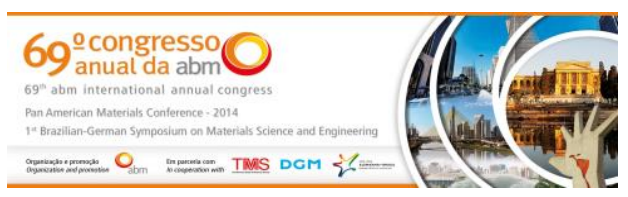

pode ser também devida a erros de medição [6] e a contribuição ou sill $\left(\boldsymbol{C}_{\mathbf{1}}\right)$ : é a diferença entre o patamar $(\boldsymbol{C})$ e o efeito pepita $\left(\boldsymbol{C}_{\mathbf{0}}\right)$, que corresponde a variância a priori dos dados.

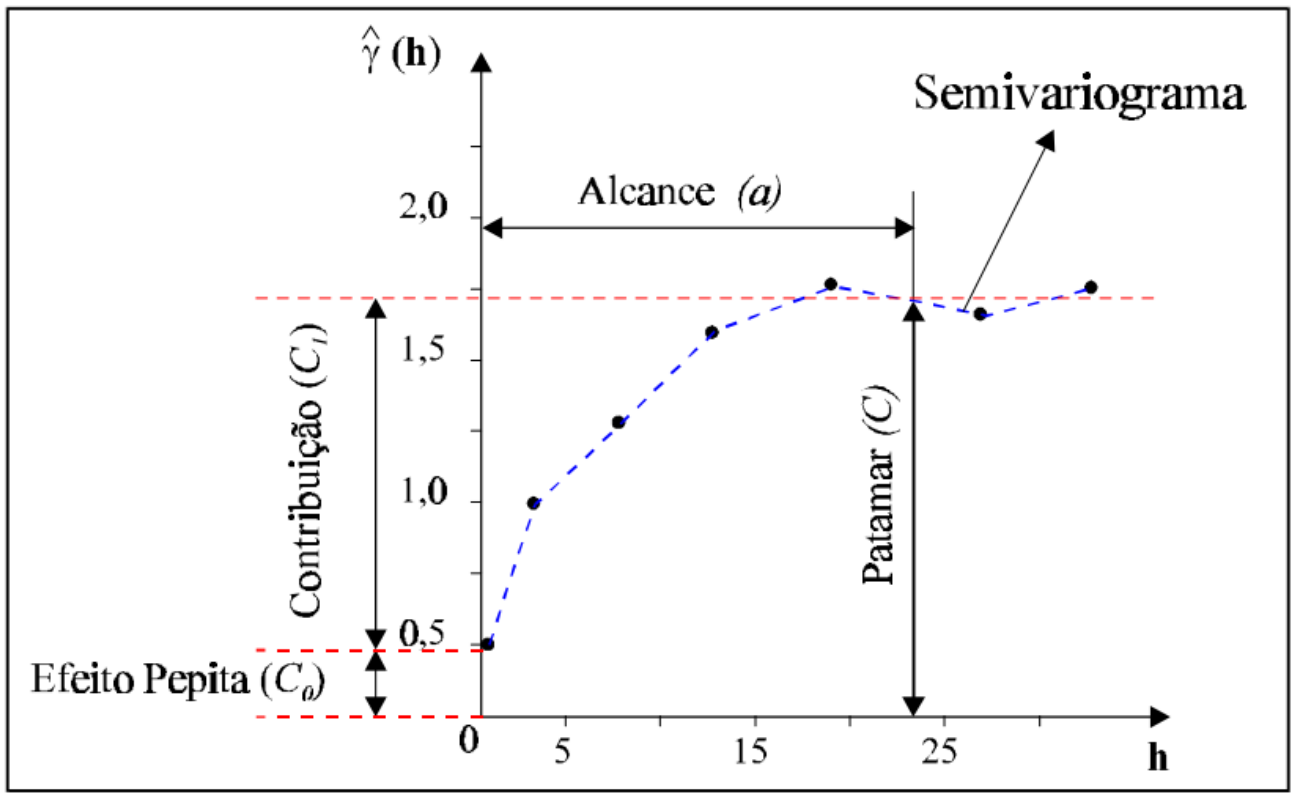

Figura 1. Componentes Semivariograma

Com a adição de erros (Tabela 1), os dados se afastam do valor esperado aumentando a variância de cada cenário e, portanto, alteram os parâmetros do modelo variográfico. Dentre eles, o mais afetado é o efeito pepita, $\boldsymbol{C}_{\mathbf{0}}$, que representa no modelo a variabilidade geológica do fenômeno e os erros referentes aos procedimentos de amostragem e protocolos de preparação inadequados. As direções de maior e menor continuidade são $157,5^{\circ}$ e $67,5^{\circ}$ e os variogramas experimentais modelados são mostrados nas Equações 3, 4, 5 e 6 [5].

$$
\begin{aligned}
& \gamma_{\mathrm{Erro}_{0} \%}(\mathrm{~h})=\mathbf{0 . 8 0}+1.0 \cdot \operatorname{Sph}(1) \cdot\left(\frac{\mathrm{N} 157.5 \mathrm{E}}{22 \mathrm{~m}}, \frac{\mathrm{N} 67.5 \mathrm{E}}{21 \mathrm{~m}}\right)+4.40 \cdot \operatorname{Sph}(2) \cdot\left(\frac{\mathrm{N} 157.5 \mathrm{E}}{71 \mathrm{~m}}, \frac{\mathrm{N} 67.5 \mathrm{E}}{42 \mathrm{~m}}\right) \\
& \gamma_{\text {Errro }_{10} \%}(h)=1.00+1.0 \cdot \operatorname{Sph}(1) \cdot\left(\frac{\mathrm{N} 157.5 \mathrm{E}}{39 \mathrm{~m}}, \frac{\mathrm{N} 67.5 \mathrm{E}}{20 \mathrm{~m}}\right)+4.40 \cdot \operatorname{Sph}(2) \cdot\left(\frac{\mathrm{N157.5E}}{75 \mathrm{~m}}, \frac{\mathrm{N} 67.5 \mathrm{E}}{41 \mathrm{~m}}\right) \\
& \gamma_{E r r o 20 \%}(h)=1.60+2.0 \cdot \operatorname{Sph}(1) \cdot\left(\frac{\mathrm{N} 157.5 \mathrm{E}}{35 \mathrm{~m}}, \frac{\mathrm{N} 67.5 \mathrm{E}}{30 \mathrm{~m}}\right)+4.40 \cdot \operatorname{Sph}(2) \cdot\left(\frac{\mathrm{N} 157.5 \mathrm{E}}{81 \mathrm{~m}}, \frac{\mathrm{N} 67.5 \mathrm{E}}{\mathbf{5 1 ~ m}}\right) \\
& \gamma_{\text {Erro }_{30} \%}(h)=2.00+1.0 \cdot \operatorname{Sph}(1) \cdot\left(\frac{\mathrm{N} 157.5 \mathrm{E}}{32 \mathrm{~m}}, \frac{\mathrm{N67.5E}}{22 \mathrm{~m}}\right)+4.50 \cdot \operatorname{Sph}(2) \cdot\left(\frac{\mathrm{N} 157.5 \mathrm{E}}{73 \mathrm{~m}}, \frac{\mathrm{N} 67.5 \mathrm{E}}{37 \mathrm{~m}}\right)
\end{aligned}
$$

Em seguida, os teores foram estimados utilizando krigagem ordinária, utilizando a mesma estratégia de busca para todos os cenários [9].

* Contribuição técnica ao 69ํ Congresso Anual da ABM - Internacional e ao 14ํㅡㄹ ENET - Encontro Nacional de Estudantes de Engenharia Metalúrgica, de Materiais e de Minas, 21 a 25 de julho de 2014 , São Paulo, SP, Brasil. 


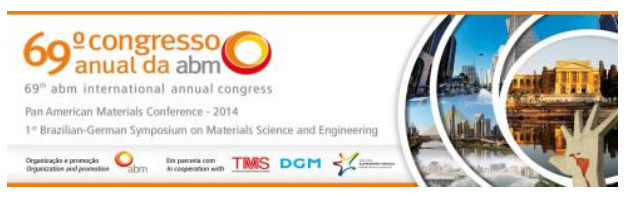

\subsection{Classificação dos Blocos}

Neste estudo de caso, iremos considerar somente o critério de teor de metal existente no bloco como informação para classificar em minério ou estéril. A técnica geoestatística empregada para as estimativas foi a krigagem ordinária [6,9], foi aplicado o teor de corte nos blocos krigados e estes foram classificados como minério ou estéril da seguinte forma:

- Se o valor estimado ( $Z^{*}$ ок) for maior que o teor de corte, foi considerado minério;

- Se o valor estimado $\left(Z^{*}\right.$ ок) for menor que o teor de corte, foi considerado estéril;

Os erros presentes nas estimativas dos blocos impactam na recuperação do metal. Se alguns blocos de minério são por algum motivo classificados como estéril, o metal será perdido. Da mesma forma, que se os blocos de material estéril forem incluídos no minério, a tonelagem total é aumentada, mas teor médio diminui (ocorre diluição).

\section{RESULTADOS E DISCUSSÃO}

A partir da estimativa foi realizada a classificação dos blocos, e definidos 5 teores de corte. Os teores de corte com valores de 0,72, 0,92, 1,23 ppm correspondem aos valores próximos aos baixos teores, o valor 2,35 que está próximo ao teor médio do depósito e o valor de 4,14 que corresponde aos teores altos. Os blocos com teores acima desses valores foram considerados minério e os blocos com valores abaixo destes foram classificados como material estéril, conforme mostrado na curva de parametrização (Figura 2). A Figura 2 mostra que a krigagem ordinária gerou estimativas próximas a distribuição real dos blocos e que o erro adicionado aos dados interfere na classificação dos blocos em estéril ou minério e no teor médio da reserva.

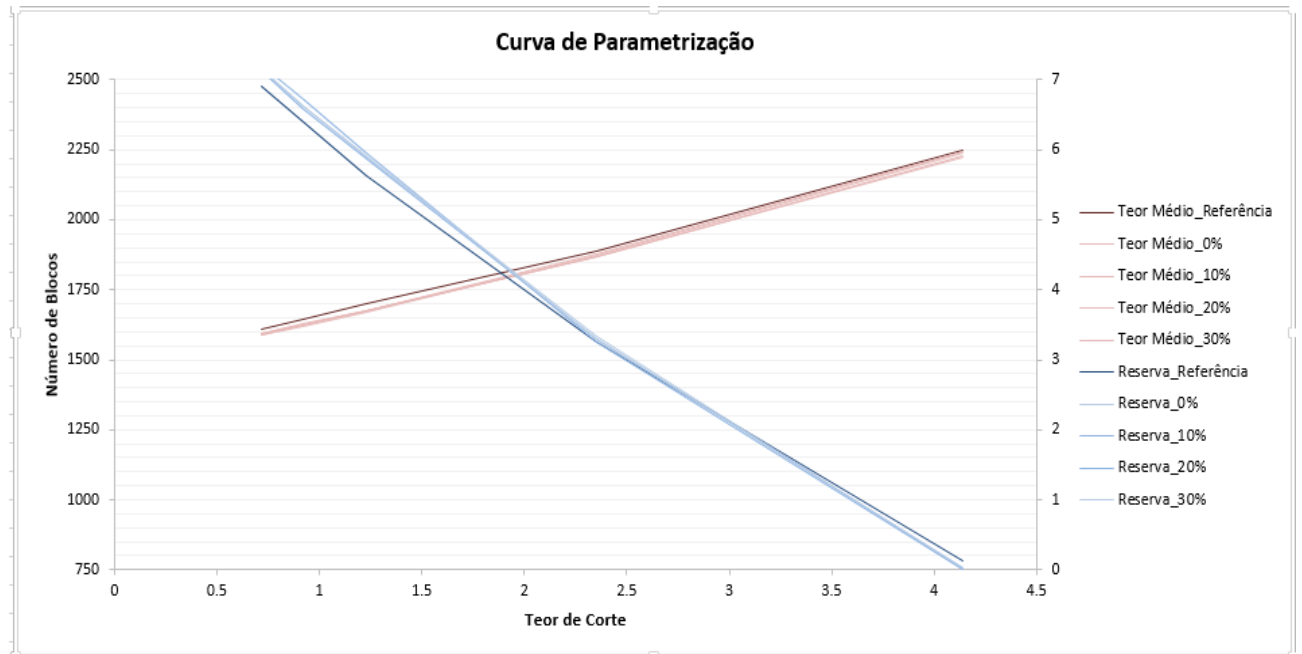

Figura 2. Curva de parametrização dos blocos com base nos dados originais com erros relativos adicionados de $10 \%, 20 \%$ e $30 \%$

As variações na curva de parametrização podem também ser avaliadas a partir das correlações existentes entre o cenário de real e a estimativa usando os dados livres e com erro de 10\%, 20\% e 30\% (Figura 3 e Tabela 2). Quanto maior o erro adicionado

* Contribuição técnica ao 69ํ Congresso Anual da ABM - Internacional e ao 14ํㅡㄹ ENET - Encontro Nacional de Estudantes de Engenharia Metalúrgica, de Materiais e de Minas, 21 a 25 de julho de 2014 , São Paulo, SP, Brasil. 


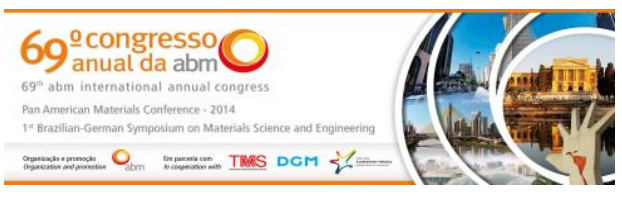

as amostras, maior a variabilidade dos dados, menor a correlação com o cenário real e o número de blocos classificados errados é maior.

Tabela 2. Correlação entre cenário de referência e as estimativas com os dados perturbados em $0 \%, 10 \%, 20 \%$ e $30 \%$

\begin{tabular}{lc}
\hline Dados & Correlação \\
\hline Erro_0\%_OK & 0,96 \\
\hline Erro_10\%_OK & 0,96 \\
\hline Erro_20\%_OK & 0,95 \\
\hline Erro_30\%_OK & 0,93 \\
\hline
\end{tabular}
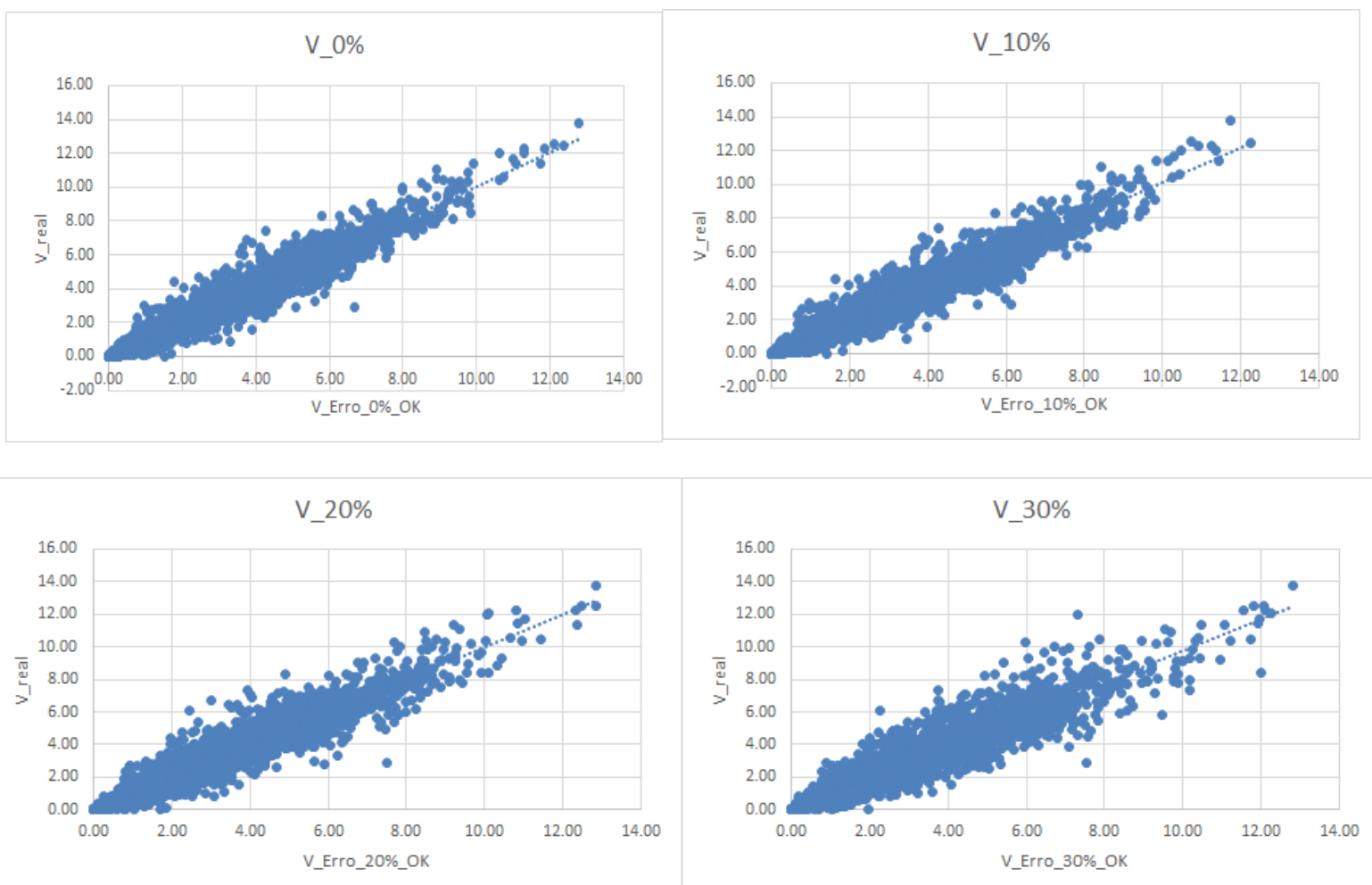

Figura 3. Scatter Plot entre os valores reais e com os dados perturbados com $0 \%, 10 \%, 20 \%, 30 \%$ estimados por KrigagemOrdinária

Para teores de corte inferiores ao teor médio do depósito $(0,72,0,92$ e 1,23 ppm), os blocos classificados como minério aumentam, o que implica no envio de maior quantidade de material estéril para a planta de beneficiamento. Desta maneira, a tonelagem de minério (ROM) é aumentada, este efeito é conhecido pela superestimativa dos teores baixos.

Para os teores de corte elevados ocorre o contrário, os blocos de minério com alto teor são subestimados, este efeito é conhecido como subestimativa dos altos teores.

* Contribuição técnica ao 69ํ Congresso Anual da ABM - Internacional e ao 14ํㅡㄹ ENET - Encontro Nacional de Estudantes de Engenharia Metalúrgica, de Materiais e de Minas, 21 a 25 de julho de 2014 , São Paulo, SP, Brasil. 


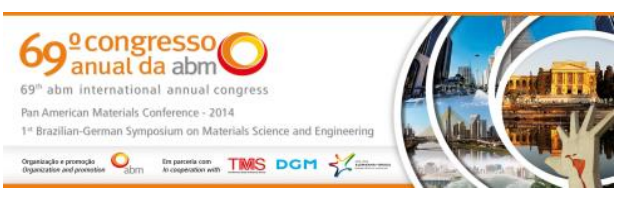

Blocos com Erro de Classificação

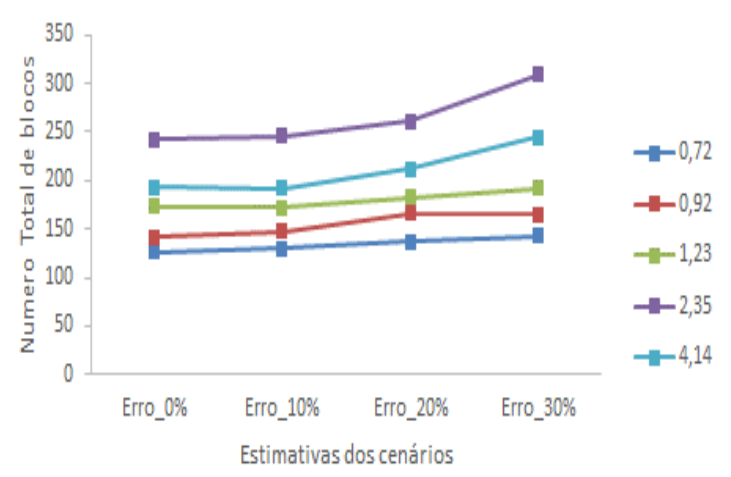

Blocos de Minério Classifficados como Estéril



Blocos de Estéril Classifficados como

Minério

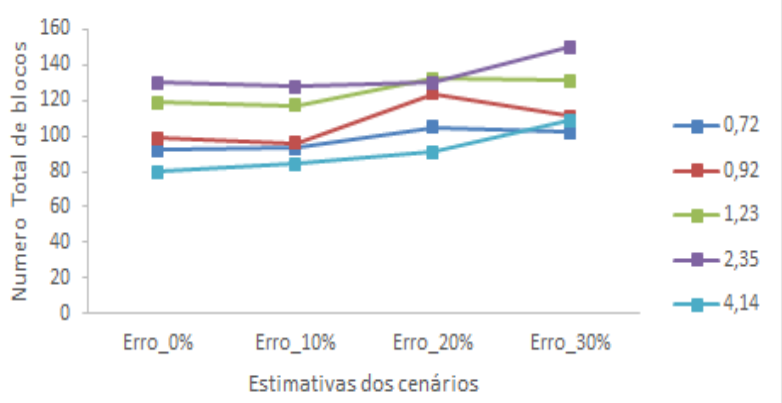

Figura 4. Erro na classificação dos blocos com os dados perturbados com erros $0 \%, 10 \%, 20 \%$ e $30 \%$.

Quanto maior o erro adicionado, maior a quantidade de blocos de minério classificados como estéril, o impacto é mais significativo. Dados com $30 \%$ de erro (Tabela 3) provocam a classificação inadequada de $4,35 \%$ dos blocos de minério, reduzindo o depósito em $14 \%$ do teor, pois o minério perdido não é recuperável.

Tabela 3. Blocos minério classificados como estéril e teor de metal descartado em diferentes cenários perturbados com $0 \%, 10 \%, 20 \%$ e $30 \%$ erro.

\begin{tabular}{cccc}
\hline $\begin{array}{c}\text { Teor } \begin{array}{c}\text { de } \\
\text { Corte }\end{array} \\
\text { Crro_0\% }\end{array}$ & Dados & $\begin{array}{c}\text { \% Blocos } \\
\text { minério } \\
\text { classificados } \\
\text { como estéril }\end{array}$ & $\begin{array}{c}\text { \% Metal } \\
\text { enviado } \\
\text { para } \\
\text { estéril }\end{array}$ \\
\hline \multirow{3}{*}{4,14} & 3,60 & 11 \\
\cline { 2 - 4 } & Erro_10\% & 3,50 & 11 \\
\cline { 2 - 4 } & Erro_20\% & 3,80 & 12 \\
\cline { 2 - 4 } & Erro_30\% & 4,35 & 14 \\
\hline
\end{tabular}

Estes exemplos demonstram o impacto do erro amostral e do protocolo de preparação nas perdas de metal, além da diluição que pode ocorrer em uma operação de mina devido a classificação errada dos blocos estimados.

Estes erros podem ter várias origens, tais como: contaminação de incrementos e das amostras, presença de material remanescente no circuito de amostragem que não

* Contribuição técnica ao 69ํ Congresso Anual da ABM - Internacional e ao 14ํㅡN Nacional de Estudantes de Engenharia Metalúrgica, de Materiais e de Minas, 21 a 25 de julho de 2014 , São Paulo, SP, Brasil. 


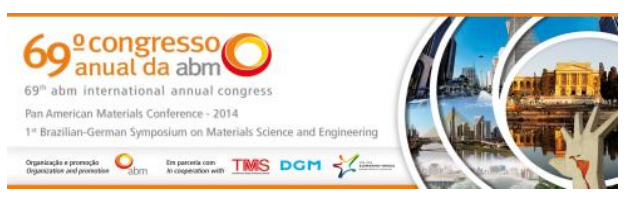

tenha sido recuperado no final de uma operação, alteração na composição química e física da amostra, dentre outros.

Independente da metodologia para a estimativa dos blocos, a combinação dos erros de amostragem e analíticos afetam diretamente no cálculo e modelagem do variograma, principalmente na determinação do efeito pepita que pode levar a suposições errôneas sobre a continuidade do minério. Portanto, estes erros devem ser evitados tanto quanto possível.

\section{CONCLUSÃO}

Problemas na amostragem e nos protocolos de preparação levam à introdução de erros aleatórios, causando a classificação incorreta do minério. Quanto maior o teor de corte, maior será a possibilidade de ocorrência de erros na classificação dos blocos. Quando o teor esperado pela produção não é obtido, o teor de corte pode ser aumentado, e devido ao erro de classificação os blocos com alto de teor de metal podem ser perdidos e reduzir as reservas de minério.

Evidenciamos que práticas de amostragem e protocolos de amostragem inadequados podem levar a um modelo variográfico impróprio e consequentemente a uma estimativa que não reproduz a realidade do depósito. Dados com erros adiciona uma componente artificial relevante que não pertence a natureza do fenômeno acarretando um engano a respeito da continuidade espacial do fenômeno. Estes procedimentos inadequados podem estar relacionados ao baixo custo operacional, a práticas de amostragem incorretas que geram valores consideráveis nas perdas. A má classificação dos blocos pode ser evitada com a amostragem coerente, um protocolo de preparação de amostras adequado e um programa de controle e monitoramento da qualidade das amostras; tais procedimentos podem evitar perdas financeiras e promover a utilização sustentável dos recursos minerais.

\section{Agradecimentos}

Ao CNPq, por ter possibilitado e financiado esta pesquisa.

Aos colegas e professores do LPM-UFRGS, Laboratório de Pesquisa Mineral e Planejamento Mineiro pelo apoio e auxilio no desenvolvimento deste trabalho.

\section{REFERÊNCIAS}

1 Chieregati AC. Reconciliação pro- ativa em empreendimentos mineiros [Tese de doutorado]. São Paulo: Escola Politécnica, Universidade de São Paulo; 2007.

2 Grigorieff A, Costa JFCL, Koppe J. O problema de amostragem manual na indústria mineral. REM: Revista Escola de Minas. 2005;55(3):229-233.

3 Grigorieff A. Desenvolvimento de um novo protocolo de amostragem de carvão mineral [Dissertação de mestrado]. Porto Alegre: Escola de Engenharia, PPGEM, UFRGS; 2002.

4 Gy P, Pitard FF. Pierre Gy's sampling theory and sampling practice: heterogeneity, sampling correctness, and statistical process control. 2nd ed. Boca Raton: CRC Press; 1989.

5 Isaaks EH, Srivastava MR. An Introduction to Applied Geostatistics. New York: Oxford University Press; 1989.

* Contribuição técnica ao 69ํ Congresso Anual da ABM - Internacional e ao 14ํㅡㄹ ENET - Encontro Nacional de Estudantes de Engenharia Metalúrgica, de Materiais e de Minas, 21 a 25 de julho de 2014 , São Paulo, SP, Brasil. 


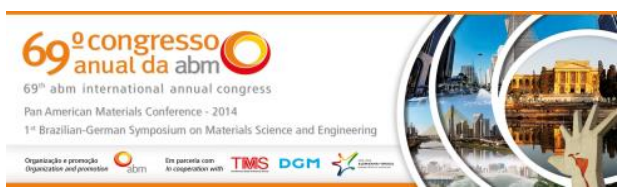

6 Matheron G. Principles of geostatistics. Economic Geology. 1963:58:11246-66.

7 Magri EV, Ortiz JC. Estimation of Economic Losses Due to poor Blast Hole Sampling in Open Pits, Proceedings of Geostat 2000 (CD-ROM) pp.1-10.

8 Goovaerts P. Geostatistics for Natural Resources Evaluation. New York: Oxford University Press; 1997.

9 Sinclair AJ, Garston HBP. Applied Mineral Inventory Estimation. Cambridge: Cambridge University Press; 2002.

* Contribuição técnica ao 69 Congresso Anual da ABM - Internacional e ao 14을 ENEMET - Encontro Nacional de Estudantes de Engenharia Metalúrgica, de Materiais e de Minas, 21 a 25 de julho de 2014, São Paulo, SP, Brasil. 